\title{
Solubilization of electrolyte solutions in AOT reversed micelles. Conductivity percolation and phase behavior
}

\author{
C. Tondre and A. Derouiche \\ Laboratoire d'Etude des Solutions Organiques et Colloïdales (L.E.S.O.C.) \\ Unité Associée au C.N.R.S. n 406, Université de Nancy I, B.P. 239 54506, Vandoeuvre-lès-Nancy Cedex, France
}

\begin{abstract}
It is well known that the amount of water that can be incorporated in AOT reversed micelles is dependent on its salinity [1] and on the nature of the electrolyte [2]. A maximum of solubility exists, which has been shown by Shah et al. [1] to result from two distinct behaviors of the surfactant film, depending on whether it is attractive (case of low salt concentration where the phase behavior is governed by a critical droplet radius $R^{c}$ ) or not (case of high salt concentration, where the phase behavior is governed by the spontaneous radius of curvature $\mathrm{R}^{\mathrm{o}}$ ). Biphasic systems separating with an excess water phase (the so-called Winsor II systems) can only be obtained in the high salt situation. The phase behavior induced by the presence of a specific electrolyte depending on its concentration is not fully understood.
\end{abstract}

\section{Introduction}

The work presented here is a contribution to a better understanding of the effect of different salts on the characteristics of the surfactant film (attractive or non-attractive) as evidenced from conductivity measurements. It was undertaken in relation with our current liquid membrane transport studies using Winsor II systems in which the microemulsion droplets play the part of mobile carriers between two liquid-liquid interfaces [3]. It was aimed at i) determining the conditions for obtaining Winsor II systems; ii) investigating the effect of changing the nature of the electrolyte; iii) looking for the possibility of percolating behavior in microemulsions leading to Winsor II systems.

From titration of AOT/decane binary mixtures with different electrolyte solutions, the electrolyte concentration at maximum water solubilization was found to be very much dependent on the nature of the salt used: the larger the electrostatic interactions between the cation and the AOT polar heads, the smaller is the amount of salt needed. Specific effects due to differences in the extent of hydration of the cations were also observed.

Whatever the nature of the salt, maximum water solubilization correlates quite well with a spec- tacular drop of conductivity right when passing the solubilization maximum. It was concluded that for a very tiny increase in water salinity around this maximum, the droplet behavior switches from attractive to non-attractive. From these observations, the possibility of percolating phenomena in the Winsor II microemulsion phase was apparently excluded for the systems under investigation.

\section{References}

1. Hou M-J, Shah DO (1987) Langmuir 3:1086

2. Leodidis EB, Hatton TA (1989) Langmuir $5: 741$

3. Derouiche A, Tondre C (1989) J Chem Soc Faraday Trans I 85:3301; Tondre C, Derouiche A (1990) J Phys Chem 94:1624
Authors' address:
Dr. C. Tondre
Laboratoire d'Etude des Solutions Organiques
et Colloïdales (L.E.S.O.C.)
Faculté des Sciences - Université de Nancy I
B.P. $\mathrm{n}^{\circ} 239$
54506 Vandoeuvre-les-Nancy Cedex, France 